\title{
Correction to: Advances in Early Detection and Diagnostic Adjuncts in Oral Cavity Cancer
}

Camile S. Farah, Nirav Bhatia, Yastira Lalla, An Vu, Keziah John, Vishal Gupta, John Baeten, Alexander Johnson, and Deepak Kademani

\section{Correction to: Chapter 9 in: M. A. Kuriakose (ed.), Contemporary Oral Oncology: Biology, Epidemiology, Etiology, and Prevention https://doi. org/10.1007/978-3-319-14911-0_9}

The original version of this chapter did not state that permission was received to reuse text from the following sources:

Efficacy of narrow band imaging for detection and surveillance of potentially malignant and malignant lesions in the oral cavity and oropharynx: A systematic review, An N.Vu, Camile S.Farah, https://www.sciencedirect.com/science/article/ abs/pii/S1368837514000384?via\%3Dihub\# (2014)

Diagnostic accuracy of Narrow Band Imaging for the detection of oral potentially malignant disorders, AN Vu, MAT Matias, CS Farah https://onlinelibrary. wiley.com/doi/abs/10.1111/odi.12315 (2015)

Assessment of a decision making protocol to improve the efficacy of VELscope ${ }^{\mathrm{TM}}$ in general dental practice: A prospective evaluation, Nirav Bhatia, Marie Anne T. Matias, Camile S. Farah https://www.sciencedirect.com/science/article/abs/pii/ S1368837514001948?via\%3Dihub (2014)

Assessment of oral mucosal lesions with autofluorescence imaging and reflectance spectroscopy, Yastira Lalla, Marie Anne T. Matias, Camile S.Farah https:// www.sciencedirect.com/science/article/abs/pii/S0002817716302641?via\%3Dihub (Aug. 2016)

The revised version of this chapter can be found at https://doi.org/10.1007/978-3-319-14911-0_9 
The authors have obtained retrospective permission to use these sources.

In addition, the authors wish to cite the following sources as they were also used to write this chapter:

Contemporary assessment and management of head and neck cancer, Camile Farah, Keziah John and Jennifer Wu (2015)

Early detection and diagnosis of oral cancer: Strategies for improvement, P.J.Ford, S.Farah https://www.sciencedirect.com/science/article/pii/S2213538313000039? via\%3Dihub (2013) 